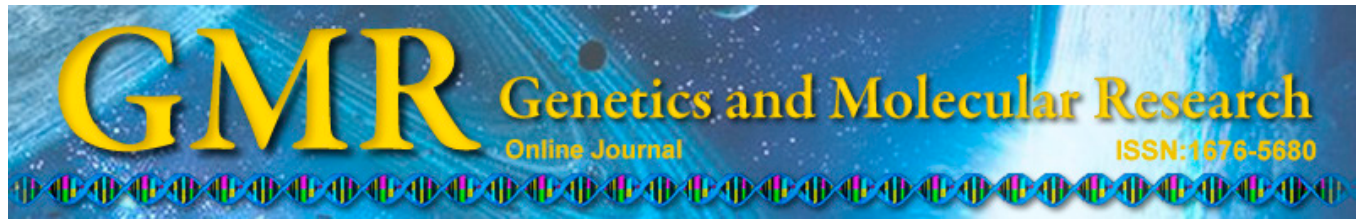

\title{
Mendelian inheritance, genetic linkage, and genotypic disequilibrium at microsatellite loci in Genipa americana L. (Rubiaceae)
}

\author{
R.O. Manoel $^{1}$, M.L.M. Freitas ${ }^{2}$, E.V. Tambarussi ${ }^{3}$, J. Cambuim ${ }^{1}$, \\ M.L.T. Moraes $^{1}$ and A.M. Sebbenn ${ }^{2}$ \\ ${ }^{1}$ Faculdade de Engenharia de Ilha Solteira, \\ Universidade Estadual Paulista "Júlio de Mesquita Filho", Ilha Solteira, \\ SP, Brasil \\ ${ }^{2}$ Instituto Florestal de São Paulo, São Paulo, SP, Brasil \\ ${ }^{3}$ Escola Superior de Agricultura "Luiz de Queiroz", \\ Universidade de São Paulo, Piracicaba, SP, Brasil \\ Corresponding author: R.O. Manoel \\ E-mail: rickom.is@gmail.com
}

Genet. Mol. Res. 14 (3): 8161-8169 (2015)

Received July 18, 2014

Accepted December 3, 2014

Published July 27, 2015

DOI http://dx.doi.org/10.4238/2015.July.27.4

\begin{abstract}
Genipa americana is a tropical tree species that is widely distributed in the humid tropical and subtropical regions of Central and South America. This study investigated Mendelian inheritance, genetic linkage, and genotypic disequilibrium at six microsatellite loci developed for G. americana. Adult trees (188) and regenerants (163) were sampled and genotyped in a fragmented population of the species. We also genotyped open-pollinated seeds from 12 seed-trees during reproductive events in 2010 and 2011. Significant deviations from the expected 1:1 Mendelian segregation were detected in $29.5 \%$ of the tests. Significant genetic linkage between pairwise loci was detected
\end{abstract}


in $54.4 \%$ of the tests, but no genotypic disequilibrium was detected between pairwise loci for adult trees and regenerants. Overall, the results indicate that the six loci analyzed may be used in studies of $G$. americana's genetic diversity and structure, its mating system, and in parentage analyses.

Key words: Conservation; Jenipapo; Microsatellites; Tropical tree species; Population genetics

\section{INTRODUCTION}

Genipa americana L. (Rubiaceae) is a dioecious tropical tree species, with pollination by bees and wasps and fruit dispersal by gravity, zoochory, and hydrochory (Carvalho, 1994). The tree is heliophytic, semideciduous, and selectively hygrophytic, and is economically important for wood and food production. The species occurs naturally in temporarily or permanently flooded areas of open forests and floodplains, in tropical and subtropical regions of Latin America (Carvalho, 1994). However, due to the intense fragmentation that its habitat has suffered, in isolated forest remnants only a few individuals of the species remain. Therefore, its genetic conservation has become a priority that only can be achieved through studies of its genetic diversity and structure, gene flow, and mating system. For these studies, microsatellite markers (simple sequence repeats, SSRs) have been used, due to their high degree of polymorphism in terms of the number of alleles (Ashley, 2010). However, if SSRs are developed for a species to be used as genetic markers, the criteria include the loci being linked and that inheritance follows Mendelian segregation rules. In addition, equilibrium linkage should be investigated as it can be altered due to selfing, correlated mating, mating between related individuals, and factors such as genetic drift and bottlenecks, which result from forest fragmentation and logging, as well as from founder events.

In the present study, we investigated Mendelian inheritance, genetic linkage, and genotypic disequilibrium at six microsatellite loci developed for G. americana by Manoel et al. (2014).

\section{MATERIAL AND METHODS}

Open-pollinated seeds were collected from the canopy of 25 seed-trees randomly selected in the Mata da Figueira, a forest fragment belonging to the Mogi Guaçu Ecological Station $\left(22^{\circ} 22^{\prime} 02^{\prime \prime} \mathrm{S}, 51^{\circ} 25^{\prime} 08^{\prime \prime} \mathrm{W}\right)$ during reproductive events in 2010 (13 seed-trees) and 2011 (12 seed-trees). The seeds were germinated separately for each seed-tree, and 27 to 80 seeds were obtained per tree. Cambial tissue from 188 adult trees and leaf tissue from 163 regenerants was also collected for genetic analysis.

DNA was extracted from the cambial tissue of all 188 adult trees using the method 
of Novaes et al. (2009). Progeny and regenerant DNA was extracted from the leaf tissue of germinated seeds using the method of Doyle and Doyle (1987).

Six primers were used (Manoel et al., 2014). Microsatellite loci were amplified by polymerase chain reaction in a $15-\mathrm{mL}$ final volume, using GoTaq ${ }^{\circledR}$ Colorless Master Mix containing $7.5 \mathrm{~mL} 2 \mathrm{X} \mathrm{GoTaq}{ }^{\circledR}$ Colorless Master Mix, $0.9 \mathrm{mM}$ of each primer (forward and reverse), $3.0 \mu \mathrm{L}$ nuclease-free water, and $7.5 \mathrm{ng}$ template DNA. The amplification program for all the primers consisted of an initial denaturing step at $94^{\circ} \mathrm{C}$ for $1 \mathrm{~min}$, followed by 35 cycles of each amplification at $94^{\circ} \mathrm{C}$ for $1 \mathrm{~min}, 1 \mathrm{~min}$ at the specific annealing temperature for each primer pair (Manoel et al., 2014), $72^{\circ} \mathrm{C}$ for $1 \mathrm{~min}$, and a final elongation step at $72^{\circ} \mathrm{C}$ for $10 \mathrm{~min}$. Amplifications were performed using a Mastercycler (Eppendorf, Hamburg, Germany). The amplification products (2-mL total reaction volume) were separated on a Fragment Analyzer ${ }^{\mathrm{TM}}$ Automated CE System [Advanced Analytical Technologies Inc. (AATI), Ames, IA, USA] using a dsDNA Reagent Kit, 35-500 bp (DNF-900, Advanced Analytical Technologies Inc.). Raw data were analyzed using the PROSize $^{\mathrm{TM}}$ (version 2.0) software (AATI).

The method developed by Gillet and Hattemer (1989) was used to investigate the Mendelian inheritance of the G. americana SSR loci, which is based on comparisons of a heterozygous maternal genotype tree with the segregation of its alleles in an openpollinated progeny. This method assumes that the loci have regular segregation and that their alleles follow classic Mendelian inheritance patterns, which are based on three main requirements: i) regular meiotic segregation during ovule production; ii) random ovule fertilization by a type of pollen; iii) no selection between the moment of fertilization and the genotyping of the seeds. The model also assumes that there is a co-dominant relationship between all of the alleles. The method further requires that the following conditions are met: all the progeny of a tree must possess a maternal allele, and in cases of heterozygous parent trees (e.g., $A_{i} A_{i}, i \neq j$ ): a) each individual offspring must possess an allele of the maternal tree, $A_{i}$ or $A_{j}$; b) the number of heterozygous progeny $A_{i} A_{j}\left(n_{i j}\right)$ must be equal to the sum of the homozygous progeny $A_{i} A_{i}\left(n_{i i}\right)$ and $A_{j} A_{j}\left(n_{j j}\right)$, or $n_{i j}=n_{i i}+n_{j j}$; and c) the number of heterozygous progeny $A_{i} A_{k}\left(n_{i k}\right)$ must equal the number of heterozygous progeny $A_{j} A_{k}\left(n_{i k}\right)$, or $n_{i k}=n_{j k}$, where $\mathrm{k} \neq \mathrm{i}, \mathrm{j}$.

Using this model and the open-pollinated progenies sampled from 25 seed-trees in two reproductive events, we proceeded to compare the segregation observed in each progeny of the heterozygous maternal tree for a given loci, with the expectation of a classic Mendelian 1:1 segregation, using a G-test (Sokal and Rohlf, 1981):

$$
G=2\left[n_{i j} \ln \left(\frac{n_{i j}}{E(n)}\right)+\left(n_{i i}+n_{i j}\right) \ln \left(\frac{\left(n_{i i}+n_{i j}\right)}{E(n)}\right)\right]
$$

where $l n$ is the natural logarithm and $E(n)$ is the expected number of genotypes for the alleles $A_{i} A_{j}\left(n_{i j}\right)$ and $A_{i} A_{i}+A_{j} A_{j}\left(n_{i i}+n_{j j}\right)$, based on $E(n)=0.5\left(n_{i j}+n_{i i}+n_{j j}\right)$ or: 


$$
G=2\left[n_{i k} \ln \left(\frac{n_{i k}}{E(n)}\right)+n_{j k} \ln \left(\frac{n_{j k}}{E(n)}\right)\right]
$$

(Equation 2)

where $\ln$ is the natural logarithm and $E(n)$ is the expected number of genotypes for the alleles $A_{i} A_{k}\left(n_{i k}\right)$ and $A_{j} A_{k}\left(n_{i k}\right)$, based on $E(n)=0.5\left(n_{i k}+n_{j k}\right)$. Additionally, a Bonferroni's correction for multiple comparisons $(95 \%, \alpha=0.05)$ was used to avoid false positives.

To test whether the loci were genetically linked, a linkage test was carried out between pairwise loci using genetic information from parent trees that were doubly heterozygous for two loci; we recorded the segregation in their progeny. In this case, the null hypothesis $\left(H_{\mathrm{O}}\right)$ was the classic Mendelian 1:1:1:1 segregation. The hypothesis of classic segregation between the pairwise loci was accepted or discarded based on a maximum likelihood G-test (Sokal and Rohlf, 1981) that was conducted for each progeny:

$$
G=2\left[n_{i k} \ln \left(\frac{n_{i k}}{E(n)}\right)+n_{i l} \ln \left(\frac{n_{i l}}{E(n)}\right)+n_{j k} \ln \left(\frac{n_{j k}}{E(n)}\right)+n_{j l} \ln \left(\frac{n_{j l}}{E(n)}\right)\right] \text { (Equation 3) }
$$

where $n_{i k}, n_{i l}, n_{j k}$, and $n_{j l}$ are the observed number of phenotypes $A_{i} B_{k}, A_{i} B_{l}, A_{j} B_{k}$, and $A_{j} B_{l}$, respectively, and $E(n)$ is the expected number of genotypes $A_{i} B_{k}, A_{i} B_{l}, A_{j} B_{k}$, and $A_{i} B_{l}$, and $\ln$ is the natural logarithm. $E(n)$ was calculated as $E(n)=0.25\left(n_{i k}+n_{i l}+n_{j k}+n_{j l}\right)$. A Bonferroni's correction for multiple comparisons $(95 \%, \alpha=0.05)$ was also applied.

A genotypic disequilibrium test was conducted for the adult trees and regenerants, since genotypic disequilibrium is expected in progeny arrays because all of the descendants always receive a maternal allele. The genotypic disequilibrium test was carried out using the FSTAT program (Goudet, 2002) that included a Bonferroni's correction $(95 \%$, $\alpha=0.05)$.

\section{RESULTS}

After the Bonferroni's correction, the results showed significant deviations from the expected 1:1 Mendelian segregation pattern in 41 cases (29.5\%) of 139 tests (Tables 1 and 2), and 74 of the 136 linkage tests (54.4\%) were significant (Table 3), suggesting that a physical linkage was present between some pairwise loci. In general, linkage occurred between different pairs of loci of different progenies, and between all of the progenies for the Gam01xGam24 pair of loci. However, after the Bonferroni's correction, no significant genotypic disequilibrium between pairs of loci in the adults and regenerants was detected, suggesting that a state of linkage equilibrium existed (Table 4). 
Table 1. Mendelian inheritance tests of three microsatellite loci of Genipa americana.

\begin{tabular}{|c|c|c|c|c|c|c|c|}
\hline Seed-tree & Genotype & $n_{1}$ & $n_{i j}: n_{i}+n_{j}$ & $G_{1}(\mathrm{GL}=1)$ & $n_{2}$ & $n_{i k}: n_{j k}$ & $G_{2}(\mathrm{GL}=1)$ \\
\hline \multicolumn{8}{|l|}{ Gam01 } \\
\hline 8 & $170 / 192$ & 75 & $6: 69$ & $62.16^{*}$ & 5 & $3: 2$ & $\mathrm{NE}$ \\
\hline 9 & $170 / 192$ & 34 & $0: 34$ & $\mathrm{NE}$ & 45 & $38: 7$ & $23.48^{*}$ \\
\hline 14 & $170 / 176$ & 44 & $13: 31$ & 7.58 & 36 & $35: 1$ & $40.77 *$ \\
\hline 21 & $170 / 192$ & 26 & $9: 17$ & 2.50 & 2 & $2: 0$ & $\mathrm{NE}$ \\
\hline 24 & $170 / 186$ & 59 & $42: 17$ & 10.93 & 18 & $12: 6$ & 2.04 \\
\hline 25 & $170 / 176$ & 32 & $12: 20$ & 2.02 & 39 & $8: 31$ & $14.49^{*}$ \\
\hline 26 & $170 / 182$ & 54 & $16: 38$ & 9.22 & 26 & $26: 0$ & $\mathrm{NE}$ \\
\hline 28 & $160 / 176$ & 33 & $10: 23$ & 5.26 & 46 & $28: 18$ & 2.19 \\
\hline 35 & $170 / 176$ & 36 & $8: 28$ & 11.76 & 44 & $43: 1$ & $51.45^{*}$ \\
\hline 37 & $160 / 170$ & 47 & $16: 31$ & 4.87 & 26 & $1: 25$ & $27.57^{*}$ \\
\hline 38 & $170 / 192$ & 37 & $1: 36$ & $42.09^{*}$ & 43 & 43:0 & $\mathrm{NE}$ \\
\hline 39 & $170 / 176$ & 27 & $1: 26$ & $28.88^{*}$ & 44 & $27: 17$ & 2.29 \\
\hline 79 & $160 / 176$ & 59 & $18: 41$ & 9.21 & 16 & $7: 9$ & 0.25 \\
\hline 85 & $170 / 186$ & 24 & $7: 17$ & 4.29 & 55 & $27: 28$ & 0.02 \\
\hline 107 & $142 / 160$ & 37 & $13: 24$ & 3.32 & 21 & $12: 9$ & 0.43 \\
\hline 128 & $160 / 172$ & 43 & $31: 12$ & 8.69 & 31 & $2: 29$ & $28.14 *$ \\
\hline 129 & $156 / 172$ & 52 & $32: 20$ & 2.79 & 7 & $3: 4$ & $\mathrm{NE}$ \\
\hline 145 & $170 / 176$ & 42 & $22: 20$ & 0.10 & 13 & $1: 12$ & 10.97 \\
\hline 147 & $160 / 176$ & 47 & $20: 27$ & 1.05 & 26 & $1: 25$ & $27.57^{*}$ \\
\hline 158 & $160 / 176$ & 39 & $23: 16$ & 1.26 & 19 & $4: 15$ & 6.78 \\
\hline 161 & $176 / 196$ & 36 & $12: 24$ & 4.08 & 29 & $26: 3$ & $20.91^{*}$ \\
\hline \multicolumn{8}{|l|}{ Gam02 } \\
\hline 8 & $164 / 180$ & 76 & $28: 48$ & 5.33 & 4 & $3: 1$ & $\mathrm{NE}$ \\
\hline 9 & $164 / 180$ & 74 & $30: 44$ & 2.66 & 4 & $2: 2$ & NE \\
\hline 14 & $188 / 208$ & 76 & $43: 33$ & 1.32 & 0 & $0: 0$ & NE \\
\hline 21 & $164 / 180$ & 25 & $14: 11$ & 0.36 & 5 & $2: 3$ & $\mathrm{NE}$ \\
\hline 24 & $164 / 180$ & 31 & $14: 17$ & 0.29 & 45 & $19: 26$ & 1.09 \\
\hline 25 & $158 / 180$ & 76 & $48: 28$ & 5.33 & 3 & $2: 1$ & $\mathrm{NE}$ \\
\hline 26 & $180 / 226$ & 61 & $39: 22$ & 4.80 & 18 & $10: 8$ & 0.22 \\
\hline 29 & $164 / 208$ & 49 & $26: 23$ & 0.18 & 31 & $4: 27$ & $19.13^{*}$ \\
\hline 37 & $162 / 180$ & 57 & $33: 24$ & 1.43 & 21 & $0: 21$ & NE \\
\hline 39 & $164 / 188$ & 57 & $26: 31$ & 0.44 & 22 & $14: 08$ & 1.66 \\
\hline 79 & $164 / 188$ & 56 & $37: 19$ & 5.89 & 20 & $0: 20$ & $\mathrm{NE}$ \\
\hline 107 & $164 / 180$ & 70 & $43: 27$ & 3.69 & 7 & $5: 2$ & $\mathrm{NE}$ \\
\hline 128 & $164 / 180$ & 71 & $37: 34$ & 0.13 & 9 & $6: 3$ & $\mathrm{NE}$ \\
\hline 129 & $158 / 174$ & 67 & $30: 37$ & 0.73 & 9 & $6: 3$ & $\mathrm{NE}$ \\
\hline 131 & $158 / 180$ & 51 & $32: 19$ & 3.35 & 15 & $11: 4$ & 3.40 \\
\hline 145 & $174 / 188$ & 40 & $12: 28$ & 6.58 & 18 & $5: 13$ & 3.68 \\
\hline 147 & $176 / 188$ & 27 & $3: 24$ & $18.59^{*}$ & 51 & $23: 28$ & 0.49 \\
\hline 158 & $164 / 188$ & 44 & $16: 28$ & 3.31 & 17 & $6: 11$ & 1.49 \\
\hline 161 & $180 / 202$ & 66 & $13: 53$ & $26.00^{*}$ & 11 & 11:0 & NE \\
\hline \multicolumn{8}{|l|}{ Gam11 } \\
\hline 8 & $162 / 172$ & 76 & $57: 19$ & $19.88^{*}$ & 3 & $2: 1$ & $\mathrm{NE}$ \\
\hline 9 & $172 / 182$ & 70 & $44: 26$ & 4.68 & 9 & $6: 3$ & $\mathrm{NE}$ \\
\hline 16 & $220 / 228$ & 67 & $30: 37$ & 0.73 & 13 & $7: 6$ & 0.08 \\
\hline 24 & $220 / 266$ & 74 & $13: 61$ & $33.80^{*}$ & 5 & $5: 0$ & NE \\
\hline 28 & $220 / 228$ & 78 & $31: 47$ & 3.30 & 1 & $1: 0$ & NE \\
\hline 35 & $186 / 196$ & 59 & $22: 37$ & 3.86 & 20 & $16: 4$ & 7.71 \\
\hline 38 & $182 / 190$ & 78 & $34: 44$ & 1.28 & 2 & $2: 0$ & $\mathrm{NE}$ \\
\hline 39 & $220 / 228$ & 76 & $27: 49$ & 6.46 & 1 & $1: 0$ & $\mathrm{NE}$ \\
\hline
\end{tabular}

$n_{1}$ and $n_{2}$, sample size; $G_{1}$ and $G_{2}$, maximum likelihood $\mathrm{G}$ statistics for the hypotheses $n_{i j}=n_{i i}+n_{i j}$ and $n_{i k}: n_{j k}$, respectively. *Significant after a Bonferroni's correction for $\alpha=0.05\left(\chi^{2}=12.83\right)$; NE, not estimated. 
Table 2. Mendelian inheritance tests of three microsatellite loci of Genipa americana.

\begin{tabular}{|c|c|c|c|c|c|c|c|}
\hline Seed-tree & Genotype & $n_{1}$ & $n_{i j}: n_{i i}+n_{i j}$ & $G_{1}(\mathrm{GL}=1)$ & $n_{2}$ & $n_{i k}: n_{j k}$ & $G_{2}(\mathrm{GL}=1)$ \\
\hline \multicolumn{8}{|l|}{ Gam06 } \\
\hline 9 & $142 / 144$ & 33 & $8: 25$ & 9.19 & 46 & $1: 45$ & $54.13^{*}$ \\
\hline 16 & $154 / 158$ & 44 & $31: 13$ & 7.58 & 36 & $13: 23$ & 2.81 \\
\hline 24 & $142 / 144$ & 46 & $37: 9$ & $18.29^{*}$ & 31 & $0: 31$ & $\mathrm{NE}$ \\
\hline 25 & $144 / 152$ & 25 & $16: 9$ & 1.99 & 48 & $7: 41$ & $26.66^{*}$ \\
\hline 26 & $144 / 152$ & 65 & $55: 10$ & $34.30^{*}$ & 13 & $13: 0$ & $\mathrm{NE}$ \\
\hline 28 & $142 / 144$ & 27 & $4: 23$ & $14.77^{*}$ & 52 & $40: 12$ & $15.91 *$ \\
\hline 29 & $142 / 144$ & 66 & $32: 34$ & 0.06 & 14 & $0: 14$ & $\mathrm{NE}$ \\
\hline 35 & $144 / 154$ & 45 & $26: 19$ & 1.09 & 35 & $0: 35$ & $\mathrm{NE}$ \\
\hline 37 & $144 / 172$ & 50 & $6: 44$ & $32.62 *$ & 24 & $15: 9$ & 1.52 \\
\hline 38 & $142 / 144$ & 74 & $47: 27$ & 5.47 & 6 & $5: 1$ & $\mathrm{NE}$ \\
\hline 79 & $142 / 144$ & 14 & $7: 7$ & 0.00 & 57 & $0: 57$ & $\mathrm{NE}$ \\
\hline 85 & $152 / 158$ & 80 & $16: 64$ & $30.84^{*}$ & 0 & $0: 0$ & $\mathrm{NE}$ \\
\hline 129 & $142 / 144$ & 46 & $24: 22$ & 0.09 & 21 & $0: 21$ & $\mathrm{NE}$ \\
\hline 131 & $144 / 152$ & 39 & $19: 20$ & 0.03 & 26 & $24: 2$ & $21.94 *$ \\
\hline 145 & $144 / 152$ & 40 & $16: 24$ & 1.61 & 8 & $3: 5$ & $\mathrm{NE}$ \\
\hline 147 & $142 / 144$ & 53 & $6: 47$ & $36.04 *$ & 7 & $0: 7$ & $\mathrm{NE}$ \\
\hline \multicolumn{8}{|l|}{ Gam24 } \\
\hline 1 & $140 / 160$ & 67 & $39: 28$ & 1.81 & 13 & $12: 1$ & 10.97 \\
\hline 9 & $140 / 148$ & 43 & $1: 42$ & $50.11 *$ & 36 & $19: 17$ & 0.11 \\
\hline 24 & $140 / 148$ & 55 & $34: 21$ & 3.10 & 24 & $2: 22$ & $19.50^{*}$ \\
\hline 35 & $140 / 148$ & 41 & $8: 33$ & $16.36^{*}$ & 38 & $28: 10$ & 8.88 \\
\hline 38 & $140 / 160$ & 57 & $15: 42$ & $13.31 *$ & 22 & $21: 1$ & $22.36^{*}$ \\
\hline 39 & $140 / 160$ & 54 & $4: 50$ & $46.34^{*}$ & 25 & $22: 3$ & $16.31^{*}$ \\
\hline 79 & $140 / 152$ & 60 & $16: 44$ & $13.59^{*}$ & 20 & $12: 8$ & 0.81 \\
\hline 85 & $134 / 148$ & 23 & $8: 15$ & 2.16 & 57 & $54: 3$ & $55.51^{*}$ \\
\hline 128 & $140 / 152$ & 68 & $10: 58$ & $37.48^{*}$ & 11 & $9: 2$ & 4.82 \\
\hline 131 & $148 / 152$ & 47 & $38: 9$ & $19.25^{*}$ & 21 & $10: 11$ & 0.05 \\
\hline 145 & $140 / 160$ & 54 & $24: 30$ & 0.67 & 8 & $6: 2$ & $\mathrm{NE}$ \\
\hline 147 & $140 / 160$ & 74 & $33: 41$ & 0.87 & 5 & $4: 1$ & $\mathrm{NE}$ \\
\hline \multicolumn{8}{|l|}{ Gam41 } \\
\hline 8 & $198 / 208$ & 77 & $31: 46$ & 2.94 & 3 & $3: 0$ & $\mathrm{NE}$ \\
\hline 9 & $198 / 208$ & 38 & $14: 24$ & 2.66 & 41 & $29: 12$ & 7.27 \\
\hline 14 & $198 / 208$ & 35 & $20: 15$ & 0.72 & 45 & $19: 26$ & 1.09 \\
\hline 16 & $198 / 208$ & 35 & $20: 15$ & 0.72 & 45 & $19: 26$ & 1.09 \\
\hline 21 & $166 / 174$ & 29 & $13: 16$ & 0.31 & 1 & $0: 1$ & $\mathrm{NE}$ \\
\hline 24 & $198 / 208$ & 78 & $42: 36$ & 0.46 & 1 & $0: 1$ & $\mathrm{NE}$ \\
\hline 25 & $198 / 208$ & 65 & $27: 38$ & 1.87 & 4 & $3: 1$ & $\mathrm{NE}$ \\
\hline 26 & $198 / 208$ & 76 & $32: 44$ & 1.90 & 4 & $2: 2$ & $\mathrm{NE}$ \\
\hline 28 & $198 / 208$ & 48 & $19: 29$ & 2.10 & 32 & $30: 2$ & $29.40^{*}$ \\
\hline 29 & $198 / 208$ & 72 & $13: 59$ & $31.81^{*}$ & 8 & $5: 3$ & $\mathrm{NE}$ \\
\hline 37 & $198 / 208$ & 63 & $29: 34$ & 0.40 & 16 & 10:6 & 1.01 \\
\hline 38 & $198 / 216$ & 53 & $14: 39$ & $12.27 *$ & 27 & $19: 8$ & 4.61 \\
\hline 39 & $198 / 208$ & 66 & $22: 44$ & 7.48 & 12 & $6: 6$ & $\mathrm{NE}$ \\
\hline 107 & $158 / 166$ & 42 & $7: 35$ & $20.38^{*}$ & 31 & $12: 19$ & 1.59 \\
\hline 129 & $198 / 208$ & 75 & $28: 47$ & 4.87 & 1 & $0: 1$ & $\mathrm{NE}$ \\
\hline 147 & $198 / 208$ & 71 & $48: 23$ & 8.99 & 5 & $0: 5$ & $\mathrm{NE}$ \\
\hline 158 & $198 / 208$ & 52 & $14: 38$ & 11.51 & 9 & $6: 3$ & $\mathrm{NE}$ \\
\hline
\end{tabular}

$n_{1}$ and $n_{2}$, sample size; $G_{1}$ and $G_{2}$, maximum likelihood $\mathrm{G}$ statistics for the hypotheses $n_{i j}=n_{i i}+n_{i j}$ and $n_{i k}: n_{j k}$, respectively. *Significant after a Bonferroni's correction for $\alpha=0.05\left(\chi^{2}=12.83\right)$; NE, not estimated. 
Table 3. Maximum likelihood G-test for the hypothesis of independent segregation between pairwise loci (1:1:1:1) of Genipa americana.

\begin{tabular}{|c|c|c|c|c|c|c|c|}
\hline Seed-tree & G & Seed-tree & G & Seed-tree & G & Seed-tree & G \\
\hline Gam01xGam02 & & Gam01xGam24 & & Gam02xGam24 & & Gam06xGam41 & \\
\hline 8 & $84.61 *$ & 24 & $26.53^{*}$ & 9 & 11.90 & 9 & $29.81^{*}$ \\
\hline 14 & $47.96^{*}$ & 35 & $87.54 *$ & 24 & $28.81^{*}$ & 16 & 0.54 \\
\hline 21 & 13.48 & 38 & $154.45^{*}$ & 39 & $53.85^{*}$ & 24 & $28.00^{*}$ \\
\hline 24 & $25.76^{*}$ & 39 & $48.11^{*}$ & 79 & $29.28^{*}$ & 25 & 16.05 \\
\hline 25 & $24.19^{*}$ & 79 & $20.66^{*}$ & 128 & $76.20 *$ & 26 & 6.08 \\
\hline 26 & $44.72 *$ & 85 & $63.31 *$ & 131 & 1.15 & 28 & $36.85^{*}$ \\
\hline 37 & $71.75^{*}$ & 128 & $57.80 *$ & 147 & 19.01 & 29 & 12.46 \\
\hline 39 & 12.03 & Gam01xGam41 & & Gam02xGam41 & & 37 & 15.04 \\
\hline 79 & 14.02 & 8 & $84.70^{*}$ & 8 & $34.98^{*}$ & 38 & 16.44 \\
\hline 107 & 2.88 & 14 & $59.53 *$ & 9 & 13.41 & 129 & $32.80 *$ \\
\hline 128 & 16.59 & 21 & 12.80 & 14 & 2.33 & 147 & $21.20^{*}$ \\
\hline 129 & 8.89 & 24 & $21.98^{*}$ & 21 & 2.74 & 158 & 14.48 \\
\hline 145 & 15.76 & 25 & 18.04 & 24 & 12.22 & Gam11xGam24 & \\
\hline 147 & 12.58 & 26 & $49.09 *$ & 25 & 8.71 & 9 & 5.00 \\
\hline 158 & 9.86 & 28 & 7.85 & 26 & 7.24 & 24 & $95.72 *$ \\
\hline 161 & $78.47 *$ & 37 & $42.71^{*}$ & 29 & 7.64 & 35 & $38.08^{*}$ \\
\hline Gam01xGam06 & & 38 & $120.61^{*}$ & 37 & $34.70^{*}$ & 38 & $81.14 *$ \\
\hline 24 & $19.82 *$ & 39 & 3.70 & 39 & 3.94 & 39 & $64.63^{*}$ \\
\hline 25 & $23.49 *$ & 107 & 5.35 & 107 & 8.44 & Gam11xGam41 & \\
\hline 26 & $65.98 *$ & 129 & 4.87 & 129 & $20.57^{*}$ & 8 & 19.45 \\
\hline 28 & 15.89 & 147 & $21.85^{*}$ & 147 & 7.88 & 9 & 2.62 \\
\hline 35 & $113.74^{*}$ & 158 & 4.53 & 158 & 8.24 & 16 & 8.21 \\
\hline 37 & $37.48 *$ & Gam02xGam06 & & Gam06xGam11 & & 24 & $85.30 *$ \\
\hline 38 & $166.65^{*}$ & 9 & $67.61^{*}$ & 9 & $38.62 *$ & 28 & 17.49 \\
\hline 85 & 10.78 & 24 & 10.50 & 16 & 13.89 & 38 & $19.67^{*}$ \\
\hline 129 & $27.50 *$ & 25 & $26.98 *$ & 24 & $83.93 *$ & 39 & 14.99 \\
\hline 145 & 13.18 & 26 & 13.20 & 28 & $42.94 *$ & Gam24xGam41 & \\
\hline 147 & $32.76^{*}$ & 29 & 6.42 & 35 & $41.62 *$ & 9 & 4.69 \\
\hline 158 & 16.56 & 37 & $20.19^{*}$ & 38 & $26.17^{*}$ & 24 & $39.56^{*}$ \\
\hline Gam01xGam11 & & 129 & $33.69 *$ & Gam06xGam24 & & 38 & $51.59 *$ \\
\hline 8 & $81.03 *$ & 131 & 18.91 & 9 & $79.44 *$ & 39 & $46.12 *$ \\
\hline 24 & $75.58^{*}$ & 145 & 19.21 & 24 & $39.02 *$ & 147 & $25.51^{*}$ \\
\hline 28 & 15.76 & 147 & 10.04 & 35 & $29.49^{*}$ & & \\
\hline 35 & $90.73 *$ & 158 & 9.28 & 38 & $67.40^{*}$ & & \\
\hline 39 & 18.31 & Gam02xGam11 & & 85 & $74.62 *$ & & \\
\hline Gam01xGam24 & & 8 & 13.79 & 131 & 18.78 & & \\
\hline 39 & $48.11^{*}$ & 9 & 10.93 & 147 & $20.91^{*}$ & & \\
\hline 79 & $20.66^{*}$ & 24 & $53.78^{*}$ & & & & \\
\hline 128 & $57.80 *$ & 39 & $22.46^{*}$ & & & & \\
\hline
\end{tabular}

*Significant after a Bonferroni's correction for $\alpha=0.05,0.00037$ ( $\left.\chi^{2}=19.61\right)$; G, G-test with three degrees of freedom.

Table 4. Genotypic disequilibrium between pairwise microsatellite loci in adult trees and regenerants of Genipa americana.

\begin{tabular}{lcc}
\hline Pairwise loci & Adult trees & Regenerants \\
\hline Gam01xGam02 & 0.10000 & 0.56875 \\
Gam01xGam06 & 0.09018 & 0.69554 \\
Gam01xGam11 & 0.10446 & 0.00893 \\
Gam01xGam24 & 0.22857 & 0.00804 \\
Gam01xGam41 & 0.21875 & 0.05893 \\
Gam02xGam06 & 0.28571 & 0.00179 \\
Gam02xGam11 & 0.86607 & 0.06607 \\
Gam02xGam24 & 0.58304 & 0.32143 \\
Gam02xGam41 & 0.72232 & 0.64018 \\
Gam06xGam11 & 0.46696 & 0.48214 \\
Gam06xGam24 & 0.74018 & 0.08036 \\
Gam06xGam41 & 0.11161 & 0.00268 \\
Gam11xGam24 & 0.58571 & 0.11607 \\
Gam11xGam41 & 0.58571 & 0.00179 \\
Gam24xGam41 & 0.47232 & 0.15982 \\
\hline
\end{tabular}

The values represent the probability of genotypic disequilibrium after 1200 permutations of alleles among individuals; after Bonferroni's corrections, $\mathrm{P}=0.00089(\alpha=0.05)$. 


\section{DISCUSSION}

Overall, the results show that all six loci segregated according to the Mendelian rules of 1:1. Deviations were detected in some progenies, but these were not repeated in all of the progenies at the same locus, indicating that the observed deviations could be attributed to sample drift, considering that the fruits contained many seeds $(>200)$ and were sampled at the maximum of 80 seeds per seed-tree. Moreover, deviations occurred in only three cases simultaneously for both realized tests $\left(n_{i j}: n_{i i}+n_{i j}\right.$ and $\left.n_{i k}: n_{j k}\right)$, suggesting that the observed deviations were random and that these loci segregate according to Mendelian rules. Therefore, the six molecular markers can be considered genetic markers.

The linkage observed between pairs of loci for several progenies may be a true genetic linkage, or may originate from individual locus deviations from a 1:1 Mendelian segregation. Loci with deviation from a 1:1 segregation may exhibit linkage disequilibrium. Some progenies showed deviations in some loci, and were used for the linkage tests. Therefore, segregation deviations in an individual locus may have generated the significant G-test values. For example, in 43 of the 74 linkage tests (58\%), significant linkage was present in at least in one locus, with significant deviation from a 1:1 segregation. However, for the pairwise loci Gam01xGam24, there was strong evidence of linkage, because all of the progeny exhibited significant linkage. Ideally, one of these loci should be excluded for some population genetic analyses, particularly mating system and parentage analysis studies.

Furthermore, some linkage may have occurred due to small sample sizes. Tambarussi et al. (2013) studied two populations of Cariniana legalis, and detected deviations from the null hypothesis of a 1:1:1:1 segregation between pairwise loci; the authors attribute their results to the low number of descendants used in the analysis. This may have occurred in the present study for some specific cases, because although a substantial number of descendants of each seed-tree was used (up to 80 seeds), only the seeds with both alleles of maternal origin in the two loci were included in the tests, which substantially reduced the number of descendants in the analysis. A similar result was reported by Tarazi et al. (2010), who studied 20 samples per progeny collected from 28 Copaifera langsdorffi seed-trees. Furthermore, the observed deviations from the null hypothesis for the segregation of individual loci, and for the pairwise loci, may have been indirectly caused by prezygotic effects, such as meiotic drift, differential gametic viability, differential reproductive success (gametic incompatibility); or by postzygotic mechanisms, such as inbreeding depression due to natural selection (Hufford and Hamrick, 2003), particularly because seedlings were analyzed, which represent a post-fertilization stage. Pre- and postzygotic effects were suggested by Tarazi et al. (2010) as the causes of the deviations found in their study on C. langsdorffii.

Genotypic disequilibrium in molecular markers is one of the basic assumptions for their use in studies of genetic diversity and structure, mating systems, and parentage analyses. We found no evidence of genotypic disequilibrium between pairs of loci in adults and regenerants, suggesting linkage equilibrium. This result is also an indication that the observed deviations in the linkage tests may have been caused by sample drift.

The six loci exhibited Mendelian inheritance, genotypic equilibrium, and were not linked, with the exception of the pairwise loci Gam01xGam24. Therefore, we suggest excluding Gam01 and Gam24 from population genetic analyses, in particular from mating system and parentage analysis studies. 


\section{ACKNOWLEDGMENTS}

Research supported by Fundação de Amparo à Pesquisa do Estado da São Paulo (FAPESP; \#2010/19613-4) and Conselho Nacional de Desenvolvimento Científico e Tecnológico (CNPq; \#473677/2010-5). The authors would like to thank FAPESP for financial support provided to R.O. Manoel (scholarship \#2011/01518-8).

\section{REFERENCES}

Ashley MV (2010). Plant parentage, pollination, and dispersal: How DNA microsatellites have altered the landscape. Crit. Rev. Plant Sci. 29: 148-161.

Carvalho PER (1994). Espécies Florestais Brasileiras: Recomendações Silviculturais, Potencialidades e Uso da Madeira. EMBRAPA-CNPF, Colombo.

Doyle JJ and Doyle JL (1987). A rapid DNA isolation procedure for small quantities of fresh leaf tissue. Phytochemistry 19: 11-15.

Gillet E and Hattemer HH (1989). Genetic analysis of isoenzyme phenotypes using single tree progenies. Heredity 63: 135-141.

Goudet J (2002). FSTAT (Version 2.9.3.2.): a computer program to calculate F-statistics. J. Hered. 86: 485-486.

Hufford KM and Hamrick JL (2003).Viability selection at three early life stages of the tropical tree Platypodium elegans (Fabaceae, Papilionoideae). Evolution 57: 518-526.

Manoel RO, Freitas MLM, Barreto MA, Moraes MLT, et al. (2014). Development and characterization of 32 microsatellite loci in Genipa americana (Rubiaceae). Appl. Plant Sci. 2: 1300084.

Novaes RML, Rodrigues JG and Lovato MB (2009). An efficient protocol for tissue sampling and DNA isolation from the stem bark of Leguminosae trees. Genet. Mol. Res. 8: 86-96.

Sokal RR and Rohlf FJ (1981). Biometry: the principles and practice of statistics in biological research. MacMillan, New York.

Tambarussi EV, Vencovsky R, Freitas MLM and Sebbenn AM (2013). Mendelian inheritance, genetic linkage, and genotypic disequilibrium at nine microsatellite loci of Cariniana legalis (Mart.) O. Kuntze. Genet. Mol. Res. 12: $5442-5457$.

Tarazi R, Sebbenn AM, Mollinari M and Vencovsky R (2010). Mendelian inheritance, linkage and linkage disequilibrium in microsatellite loci of Copaifera langsdorffii Desf. Conserv. Genet. Resour. 2: 201-204. 